\title{
Evaluation des différences génétiques dans l'importance et la répartition du tissu musculaire, entre 25 et 150 jours, sur des agneaux Romanov, Berrichon $\times$ Romanov, Texel $\times$ Romanov, Lacaune $\times$ Romanov, Charmois $\times$ Romanov
}

\author{
J. TEYSSIER et M. PRUD'HON \\ avec la collaboration technique de J. Angevain et J. Goussopoulos \\ I.N.R.A., Station de Physiologie animale \\ E.N.S.A., place Viala, 34060 Montpellier Cedex, France
}

Résumé

Quatre-vingts agneaux mâles doubles appartenant à 5 génotypes (Romanov, Berrichon $\times$ Romanov, Texel $\times$ Romanov, Lacaune $\times$ Romanov, Charmois $\times$ Romanov) ont été abattus à différents âges $(25,50,100$ et 150 jours $)$. Les muscles individuels de la demi-carcasse ont été pesés et regroupés en régions musculaires (10) afin d'étudier l'influence de la race des béliers sur l'importance relative et la répartition de la musculature.

Après vérification des hypothèses de base, un modèle d'analyse de covariance a été retenu pour comparer les relations d'allométrie établies, pour les différents génotypes, entre le poids total de muscle et le poids de carcasse ou entre le poids de chaque région musculaire et le poids total de muscle. Soit choisir entre trois hypothèses : droites d'allométrie non parallèles $\left(\mathrm{H}_{1}\right)$, parallèles $\left(\mathrm{H}_{2}\right)$ ou identiques $\left(\mathrm{H}_{3}\right)$.

A l'exception des muscles de la jambe $\left(\mathrm{H}_{1}\right)$, les coefficients de croissance relative sont homogènes entre génotypes $\left(\mathrm{H}_{2}\right.$ ou $\left.\mathrm{H}_{3}\right)$ dans l'intervalle des poids étudiés. A poids de carcasse égal, les croisés Berrichon $\times$ Romanov ont la musculature la moins développée et les croisés Charmois $\times$ Romanov la plus développée, respectivement 95,9 et 101,1 quand on les compare aux agneaux Romanov (100). Pour un même poids total de muscle, les différences significatives dans la répartition de la musculature (essentiellement régions musculaires de l'abdomen et de la partie antérieure de la carcasse) sont des différences dont les conséquences économiques sont minimes. Pour certaines régions, elles semblent résulter en grande partie des différences de maturité des agneaux des différents génotypes étudiés.

\section{Introduction}

Le croisement industriel est une pratique courante pour la production d'agneaux de boucherie .Il peut avoir une influence sur la croissance, le format et la qualité des carcasses (conformation, composition tissulaire, répartition des tissus...) des agneaux croisés. L'objectif de cette présente étude sur plusieurs génotypes d'agneaux mâles, issus de brebis Romanov accouplées à des béliers Romanov, Berrichon du Cher, Texel, Lacaune et Charmois, est d'évaluer l'influence de la race des béliers sur le 
poids total de muscle et la répartition en 10 régions musculaires, respectivement pour un même poids de carcasse et un même poids total de muscle. Nous essayons parallèlement de dégager les problèmes essentiels de l'utilisation de l'analyse de covariance qui est la méthode d'analyse habituellement retenue quand les animaux des génotypes comparés sont abattus à divers stades de la croissance.

De nombreux auteurs ont utilisé des protocoles d'abattage à divers stades de la croissance dans la comparaison des caractéristiques musculaires d'agneaux de différents génotypes mais leurs études sont difficilement comparables. Le choix des génotypes et les conditions d'élevage sont souvent spécifiques. Les intervalles d'étude et la définition des stades d'abattage diffèrent : abattages aux mêmes âges, entre la naissance et la maturité (FouRIE et al., 1970 ; JURY et al., 1977), aux mêmes poids entre 34 et $54 \mathrm{~kg}$ (THомpson et al., 1979 a et b), aux mêmes degrés de maturité, entre 40 et 76 p. 100 de l'estimation du poids adulte (MCClelland et al., 1976; TaYlor et al., 1980). La division de la musculature de la carcasse pour l'étude de la répartition du tissu musculaire est aussi plus ou moins fine : étude de muscles individuels (JURY et al., 1977 ; TAYLOR et al., 1980), partition de la musculature en 9 régions anatomiques par regroupement de muscles individuels (JURY et al., 1977 ; Thompson et al., $1979 \mathrm{~b}$ ) ou en cinq régions par découpe de la carcasse avant dissection (SeEbeck, 1968). Au niveau de l'analyse statistique, les travaux de McClelland et al. (1976) et de TAYLOR et al. (1980) sont les seuls où les stades d'abattage sont les modalités d'un facteur qualitatif étudié dans une analyse de variance ou de covariance. Généralement, les variations entre stades d'abattage sont prises en compte dans l'analyse statistique par l'introduction d'une covariable dans le modèle linéaire (poids de carcasse, poids de muscle de la carcasse). C'est le cas dans notre étude.

Les données sur le tissu musculaire, qui font l'objet ici d'une étude détaillée, ont été obtenues dans le cadre d'une étude plus générale portant sur les effets du croisement avec les brebis prolifiques de race Romanov (PRUD'HON et al., 1980) : étude réalisée par la Station de Physiologie animale, entre 1977 et 1980 , sur un protocole d'abattage à différents âges des agneaux croisés.

\section{Matériel et méthodes}

L'étude comparative porte sur 80 agneaux mâles nés du croisement entre des brebis de race Romanov, issues des élevages de la S.A.G.A. de Toulouse, et des béliers appartenant à 5 races (Romanov, Berrichonne du Cher, Texel, Lacaune, Charmoise). Les races des béliers ont été choisies en raison de leurs différences de format et de conformation. Les agneaux de race pure Romanov forment un échantillon de référence.

Les agneaux, exclusivement des jumeaux, ont été sevrés à 50 jours puis ils ont été élevés en bergerie, à Montpellier, à partir de fourrage sec et d'un complément (orge 70 p. 100 + pois 30 p. 100) dont la quantité était limitée à $800 \mathrm{~g}$ par jour.

Le protocole d'abattage des agneaux est donné par le tableau 1. Les stades d'abattage sont fixés par l'âge avec une fourchette de plus ou moins un jour ; l'intervalle 25-150 jours couvrant la quasi totalité des abattages pratiqués pour les différents types de production d'agneaux du marché français. Les tableaux 2 et 3 donnent les 
valeurs moyennes et les valeurs extrêmes du poids d'abattage et du poids total de muscle de la demi-carcasse disséquée. L'affectation des agneaux à un groupe d'abattage a été faite à la naissance.

\section{TABleau 1}

Nombre d'agneaux de chaque génotype abattus à chaque stade.

Number of lambs of each genotype slaughtered at each stage.

\begin{tabular}{r|c|c|c|c|c}
\hline & \multicolumn{5}{|c}{ Génotypes } \\
\cline { 2 - 6 } & Romanov & $\begin{array}{c}\text { Berrichon } \\
\times \\
\text { Romanov }\end{array}$ & $\begin{array}{c}\text { Texel } \\
\times \\
\text { Romanov }\end{array}$ & $\begin{array}{c}\text { Lacaune } \\
\times \\
\text { Romanov }\end{array}$ & $\begin{array}{c}\text { Charmois } \\
\times \\
\text { Romanov }\end{array}$ \\
\hline 25 jours $\ldots \ldots \ldots \ldots$ & 4 & 4 & 4 & 4 & 4 \\
50 jours $\ldots \ldots \ldots \ldots$ & 4 & 4 & 4 & 4 & 4 \\
100 jours $\ldots \ldots \ldots \ldots$ & 4 & 4 & 4 & 4 & 4 \\
150 jours $\ldots \ldots \ldots \ldots$ & 4 & 4 & 4 & 4 & 4 \\
\hline \hline
\end{tabular}

Les agneaux ont été abattus le matin, après une période de jeûne de 12 à 18 heures. Chaque animal a été dépecé et éviscéré. La tête et les extrémités des membres ont été séparés de façon à obtenir la carcasse classique de boucherie. Le même jour, après 6 heures à $4^{\circ} \mathrm{C}$, les dépôts de gras internes de la carcasse ont été en!evés et la dissection musculaire a été faite sur la demi-carcasse droite selon la technique décrite par BENEVENT (1968). Les muscles, prélevés et pesés individuellement, sont regroupés en 10 régions musculaires (cf. annexe et fig. 1) proches de celles définies par LOHSE et al. (1971). Nous étudions aussi deux autres ensembles de muscles qui correspondent à la composition musculaire de deux grandes régions définies par découpe de la carcasse : l'épaule et le gigot raccourci (cf. annexe et fig. 1).

\section{A. Analyse statistique}

Nous retenons un modèle d'analyse de covariance pour décrire et tester les différences, entre génotypes, dans l'évolution du poids total de muscle de la carcasse (y) par rapport au poids de la carcasse (x), puis du poids de chaque région musculaire (y) par rapport au poids total de muscle $(\mathrm{x})$.

Soit un modèle interactif (les coefficients de la covariable dépendent du génotype $i$, avec $i=1$ à 5) :

où :

$$
\mathbf{Y}_{\mathrm{ij}}=\mu+\alpha_{\mathrm{i}}+\beta_{\mathrm{i}} \mathbf{X}_{\mathrm{ij}}+\mathrm{e}_{\mathrm{ij}}
$$

$Y_{i j}$ et $X_{i j}$ : ln des valeurs des variables $y$ et $x$ observées pour le ij ième agneau,

$\alpha_{i} \quad:$ effet fixé du i ième génotype,

$\beta_{\mathrm{i}} \quad:$ coefficient de régression pour le $\mathrm{i}$ ième génotype,

$\mathrm{e}_{\mathrm{ij}} \quad$ : terme aléatoire résiduel. 

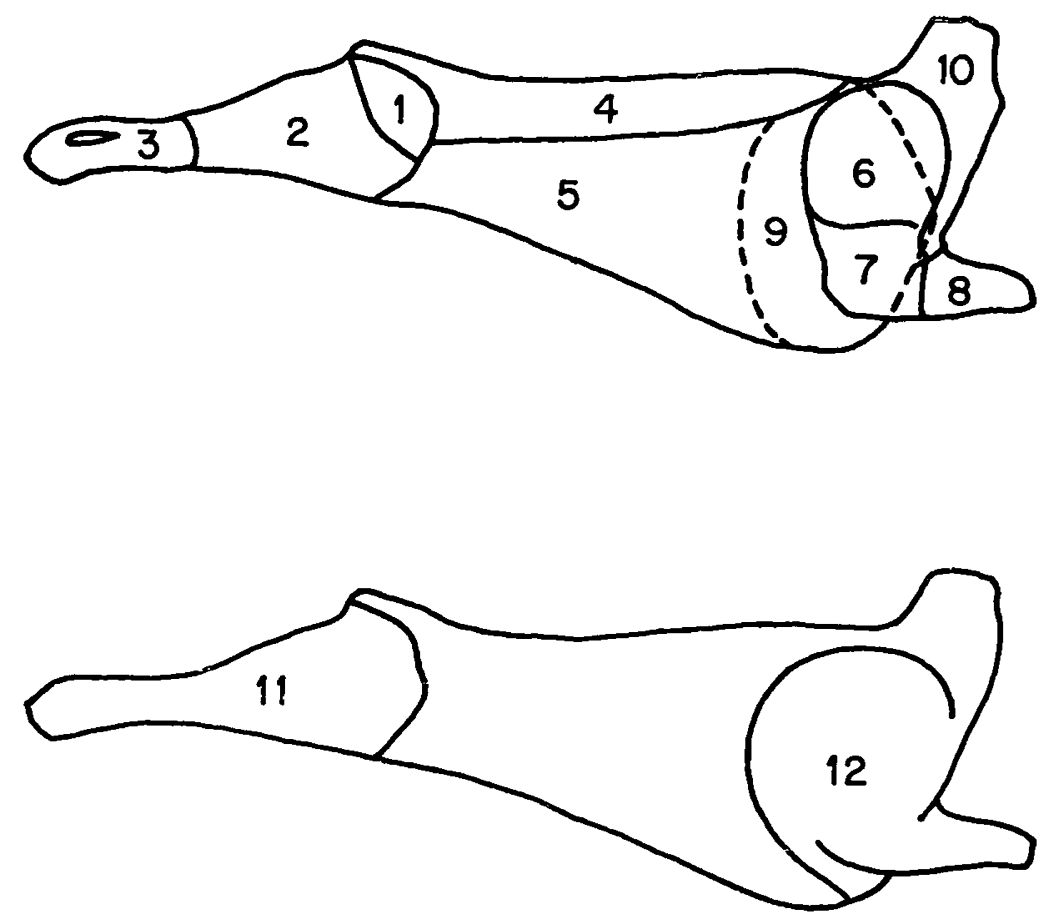

FIG. 1

Régions musculaires étudie̊es.

Studied muscle groups.

a) Bassin (1), cuisse (2), jambe (3), autour de la colonne vertébrale (4), paroi abdominale (5), épaule anatomique (6), bras (7), avant-bras (8), attache de l'épaule (9), cou (10).

b) Gigot raccourci (gi. rac.), épaule (ép.).

Ce modèle implique la linéarité des régressions calculées pour les différents génotypes et un couple de variables $\mathrm{Y}, \mathrm{X}$. Nous justifierons cette hypothèse pour chaque génotype (croissance allométrique c'est-à-dire $(\mathrm{dy} / \mathrm{ydt}) /(\mathrm{dx} / \mathrm{xdt})$ constant sur l'intervalle étudié) par calcul du carré du coefficient de corrélation linéaire $\mathrm{r}^{2} \mathrm{YX}$. Il implique aussi l'hypothèse d'homogénéité des variances résiduelles par rapport aux droites de régression (droites d'allométrie) calculées pour les différents génotypes. Cette hypothèse sera vérifiée par le test de HaRTLEY.

Sur le modèle (1) nous sommes confrontés à un problème de décision multiple : problème du choix entre 3 hypothèses $\mathrm{H}_{1}, \mathrm{H}_{2}$ et $\mathrm{H}_{3}$.

$$
\begin{aligned}
& \mathbf{H}_{1}: \mathbf{Y}_{\mathrm{ij}}=\mu+\alpha_{i}+\beta_{\mathrm{i}} \mathbf{X}_{\mathrm{ij}}+\mathrm{e}_{\mathrm{ij}} \\
& \mathbf{H}_{2}: \mathrm{Y}_{\mathrm{j} j}=\mu+\alpha_{\mathrm{i}}+\beta \mathrm{X}_{\mathrm{ij}}+\mathrm{e}^{\prime}{ }_{\mathrm{ij}} \\
& \mathrm{H}_{3}: \mathbf{Y}_{\mathrm{ij}}=\mu+\beta \mathrm{X}_{\mathrm{ij}}+\mathrm{e}^{\prime \prime}{ }_{\mathrm{ij}}
\end{aligned}
$$

Concrètement, pour un même ensemble de muscles les différents génotypes suivent : 
- des lois de croissance relative différentes si on décide $\mathrm{H}_{1}$ (droites d'allométrie non parallèles);

- une même loi de croissance relative sur des proportions initiales différentes si on décide $\mathrm{H}_{2}$ (droites d'allométrie parallèles);

- une même loi de croissance relative sur des proportions initiales identiques si on décide $\mathrm{H}_{3}$ (droites d'allométrie identiques).

Dans l'ensemble des règles de décision admissibles, on a choisi une règle de décision avec pooling de l'interaction qui présente des propriétés intéressantes décrites par Masson (1976). Cette règle de décision est très utilisée, elle est même implicite quand on accepte d'emblée l'hypothèse de parallélisme sans la tester.

Soient $\mathrm{SCE}_{1}, \mathrm{SCE}_{2}$ et $\mathrm{SCE}_{3}$, les sommes des carrés résiduelles des ajustements aux modèles (1), (2) et (3), p et $n$ le nombre de génotypes et le nombre total d'agneaux.

\section{Test 1}

On teste l'absence d'interaction entre le facteur et la covariable (test de parallélisme) en calculant la statistique du test :

\section{Test 2}

$$
F_{1}=\frac{\left(\mathrm{SCE}_{2}-\mathrm{SCE}_{1}\right) /(\mathrm{p}-1)}{\mathrm{SCE}_{1} /(\mathrm{n}-2 \mathrm{p})}
$$

Si on accepte l'hypothèse de parallélisme, on teste l'absence d'effet du facteur en présence de la covariable (test d'identité) en calculant la statistique du test :

$$
\mathrm{F}_{2}=\frac{\left(\mathrm{SCE}_{3}-\mathrm{SCE}_{2}\right) /(\mathrm{p}-1)}{\mathrm{SCE}_{2} /(\mathrm{n}-\mathrm{p}-1)}
$$

Si $F_{1}>f_{1}(5$ p. 100$)$

on décide $\mathrm{H}_{1}$, modèle (1).

Si $F_{1} \leqslant f_{1}(5$ p. 100$)$ et $F_{2}>f_{2}(5$ p. 100$)$ on décide $H_{2}$, modèle (2).

Si $F_{1} \leqslant f_{1}(5$ p. 100$)$ et $F_{2} \leqslant f_{2}\left(5\right.$ p. 100) on décide $H_{3}$, modèle (3).

$\mathrm{Si}$ on décide $\mathrm{H}_{1}$, les différences en pourcentages, entre génotypes, pour la variable $\mathrm{y}$ dépendent de la valeur de la variable $\mathrm{x}$ fixée pour la companaison. Dans ce cas, les différences n'ont pas été calculées. Si on décide $\mathrm{H}_{2}$, les différences en pourcentages sont indépendantes de la valeur de $\mathrm{x}$ fixée pour la comparaison et ont été calculées. La décision $\mathrm{H}_{3}$ implique l'absence d'effet du génotype sur l'intervalle étudié donc l'absence de différences entre génotypes.

\section{Résultats et discussion}

\section{A. Echantillonnage}

Les écarts importants observés à 150 jours entre les moyennes du poids d'abattage des différents génotypes (tabl. 2) soulignent les différences de format liées au génotype des agneaux. L'écart maximum est trouvé pour les génotypes Romanov et 
Berrichon $\times$ Romanov, respectivement $34,8 \mathrm{~kg}$ et $44,9 \mathrm{~kg}$. A cet âge, l'écart maximum entre les moyennes du poids total de muscle de la demi-carcasse est trouvé pour les génotypes Romanov et Texel $\times$ Romanov, respectivement $4,23 \mathrm{~kg}$ et $6,30 \mathrm{~kg}$ (tabl. 3).

\section{TABLEAU 2}

Valeur moyenne et valeurs extrêmes $(\mathrm{kg})$ du poids d'abattage pour chaque génotype à chaque stade d'abattage.

Mean and extreme values $(\mathrm{kg})$ of slaughter weight for each genotype at each stage of slaughter.

\begin{tabular}{|c|c|c|c|c|c|}
\hline \multirow[b]{2}{*}{ Stades d'abattage } & \multicolumn{5}{|c|}{ Génotypes } \\
\hline & Romanov & $\begin{array}{c}\text { Berrichon } \\
\times \\
\text { Romanov }\end{array}$ & $\begin{array}{c}\text { Texel } \\
\times \\
\text { Romanov }\end{array}$ & $\begin{array}{c}\text { Lacaune } \\
\times \\
\text { Romanov }\end{array}$ & $\begin{array}{c}\text { Charmois } \\
\times \\
\text { Romanov }\end{array}$ \\
\hline 25 jours & $\begin{array}{c}8,9 \\
(8,2-9,5)\end{array}$ & $\begin{array}{c}7,1 \\
(6,8-7,3)\end{array}$ & $\begin{array}{c}6,7 \\
(6,2-7,1)\end{array}$ & $\begin{array}{c}8,9 \\
(7,9-9,6)\end{array}$ & $\begin{array}{c}9,1 \\
(8,5-9,7)\end{array}$ \\
\hline 50 jours & $\begin{array}{c}14,1 \\
(11,2-16,7)\end{array}$ & $\begin{array}{c}13,5 \\
(12,6-15,8)\end{array}$ & $\begin{array}{c}14,1 \\
(12,2-17)\end{array}$ & $\begin{array}{c}15,1 \\
(: 3-16,8)\end{array}$ & $\begin{array}{c}13,8 \\
(12,7-14,5)\end{array}$ \\
\hline 100 jours & $\begin{array}{c}30,8 \\
(27,8-33,5)\end{array}$ & $\begin{array}{c}29,1 \\
(28,5-31,6)\end{array}$ & $\begin{array}{c}28,1 \\
(27-30)\end{array}$ & $\begin{array}{c}30,1 \\
(25,4-34,5)\end{array}$ & $\begin{array}{c}28,9 \\
(26,2-31,5)\end{array}$ \\
\hline 150 jours & $\begin{array}{c}34,8 \\
(31,8-39)\end{array}$ & $\begin{array}{c}44,9 \\
(40,1-49,7)\end{array}$ & $\begin{array}{c}44,1 \\
(39,6-51,9)\end{array}$ & $\begin{array}{c}38,6 \\
(37,3-41)\end{array}$ & $\begin{array}{c}35,8 \\
(33,6-37,9)\end{array}$ \\
\hline
\end{tabular}

TABLeaU 3

Valeur moyenne et valeurs extrêmes $(\mathrm{kg})$ du poids total de muscle de la demi-carcasse pour chaque génotype à chaque stade d'abattage.

Mean and extreme values $(\mathrm{kg})$ of total half carcass muscle weight for each genotype at each stage of slaushter.

\begin{tabular}{|c|c|c|c|c|c|}
\hline \multirow[b]{2}{*}{ Stades d'abattage } & \multicolumn{5}{|c|}{ Génotypes } \\
\hline & Romanov & $\begin{array}{c}\text { Berrichon } \\
\times \\
\text { Romanov }\end{array}$ & $\begin{array}{c}\text { Texel } \\
\times \\
\text { Romanov }\end{array}$ & $\begin{array}{c}\text { Lacaune } \\
\times \\
\text { Romanov }\end{array}$ & $\begin{array}{c}\text { Charmois } \\
\times \\
\text { Romanov }\end{array}$ \\
\hline 25 jours $\ldots \ldots$ & $\begin{array}{c}1,40 \\
(1,24-1,52)\end{array}$ & $\begin{array}{c}1,09 \\
(1,03-1,14)\end{array}$ & $\begin{array}{c}1,04 \\
(0,86-1,22)\end{array}$ & $\begin{array}{c}1,29 \\
(1,06-1,40)\end{array}$ & $\begin{array}{c}1,48 \\
(1,37-1,58)\end{array}$ \\
\hline 50 jours $\ldots \ldots$ & $\begin{array}{c}2,11 \\
(1,56-2,78)\end{array}$ & $\begin{array}{c}2,00 \\
(1,83-2,30)\end{array}$ & $\begin{array}{c}2,20 \\
(1,88-2,67)\end{array}$ & $\begin{array}{c}2,05 \\
(1,85-2,19)\end{array}$ & $\begin{array}{c}2,00 \\
(1,73-2,17)\end{array}$ \\
\hline 100 jours & $\begin{array}{c}4,07 \\
(3,64-4,44)\end{array}$ & $\begin{array}{c}4,20 \\
(4,15-4,22)\end{array}$ & $\begin{array}{c}4,33 \\
(4,02-4,61)\end{array}$ & $\begin{array}{c}3,87 \\
(3,32-4,50)\end{array}$ & $\begin{array}{c}4,04 \\
(3,66-4,45)\end{array}$ \\
\hline 150 jours $\ldots$ & $\begin{array}{c}4,23 \\
(3,87-4,56)\end{array}$ & $\begin{array}{c}6,08 \\
(5,78-6,52)\end{array}$ & $\begin{array}{c}6,30 \\
(5,53-7,27)\end{array}$ & $\begin{array}{c}5,08 \\
(4,75-5,50)\end{array}$ & $\begin{array}{c}4,68 \\
(4,35-5,16)\end{array}$ \\
\hline
\end{tabular}


Les contraintes expérimentales (nombre de génotypes et de stades d'abattage, dissection complète des carcasses) ont limité le nombre d'agneaux étudiés pour chaque génotype et chaque stade mais cette limite est commune aux expérimentations réunissant les mêmes contraintes. Nous avons, par contre, contrôlé des sources de variation connues (origine des mères, mode de naissance, sexe, condition d'élevage) qui augmenteraient la variabilité des résultats et nuiraient à la détection d'une influence du génotype sur un effectif trop réduit. Les poids d'abattage montrent néanmoins l'existence de biais dans l'échantillonnage des agneaux Romanov abattus à 100 et 150 jours. Les poids des agneaux Romanov abattus à 100 jours apparaissent élevés si nous nous référons, par exemple, au gain moyen quotidien 30-70 jours $(213 \mathrm{~g})$ et au poids vif à 70 jours $(16,9 \mathrm{~kg})$ rapportés par Perret \& Cournut (1975) chez des agneaux mâles doubles Romanov pour un échantillonnage sur plusieurs campagnes dans plusieurs élevages français.

\section{B. Hypothèses}

Les valeurs élevées de $\mathrm{r}^{2} \mathrm{YX}$, comprises entre 0,988 et 0,999 pour l'ensemble des régressions calculées, valident l'hypothèse de croissance allométrique sur l'intervalle d'étude quels que soient le génotype et l'ensemble de muscles étudiés. En conséquence, sur l'intervalle d'étude, nous n'avons pas envisagé d'autres ajustements que l'ajustement à une droite contrairement à d'autres auteurs qui ont retenu, pour certaines régions musculaires mais sur un intervalle plus étendu (naissance-maturité), un ajustement à deux droites de régression (LoHSE et al., 1971) ou à une régression quadratique (JURY et al., 1977). Le premier stade de notre protocole se situe après le changement d'allométrie des muscles constaté aux environs de 20 jours, dans l'intervalle naissance - 160 jours, chez des agneaux Mérinos d'Arles (BenEvent, 1971).

Les valeurs de la statistique de HartLey pour 5 génotypes ont été calculées pour tous les ensembles de muscles étudiés (tabl. 4). Quand une valeur est significative on calcule la même statistique pour 4 génotypes après élimination du génotype pour lequel on trouve une valeur extrême de la somme des carrés des écarts résiduels. L'hypothèse d'homogénéité des variances résiduelles est alors vérifiée et l'étude comparative est réalisée sur 4 génotypes (muscles de la paroi abdominale, de l'épaule anatomique et du bras).

\section{Poids total de muscle de la carcasse}

L'hypothèse de parallélisme est acceptée et 1'hypothèse d'identité (absence d'effet du génotype) est rejetée (tabl. 5). L'évolution du poids total de muscle par rapport au poids de carcasse, pour les différents génotypes, est donc décrite par une même loi de croissance relative $(0,99)$ sur des proportions initiales différentes $\left(\mathbf{H}_{2}\right)$. Exprimées en pourcentages du poids total de muscle des agneaux Romanov, les valeurs obtenues à poids de carcasse égal sont respectivement 95,9, 98,4, 100,2 et 101,1 pour les croisés Berrichon $\times$ Romanov, Lacaune $\times$ Romanov, Texel $\times$ Romanov et Charmois $\times$ Romanov (tabl. 6). Les croisés Berrichon $\times$ Romanov ont donc la musculature la moins développée et les croisés Charmois $\times$ Romanov la musculature la plus développée.

Le résultat obtenu par croisement avec les béliers Berrichon, race bouchère de grand format, ne peut être la conséquence des différences de maturité des génotypes car le coefficient de croissance relative commun est très proche de $1(0,99)$. Prud'HON 


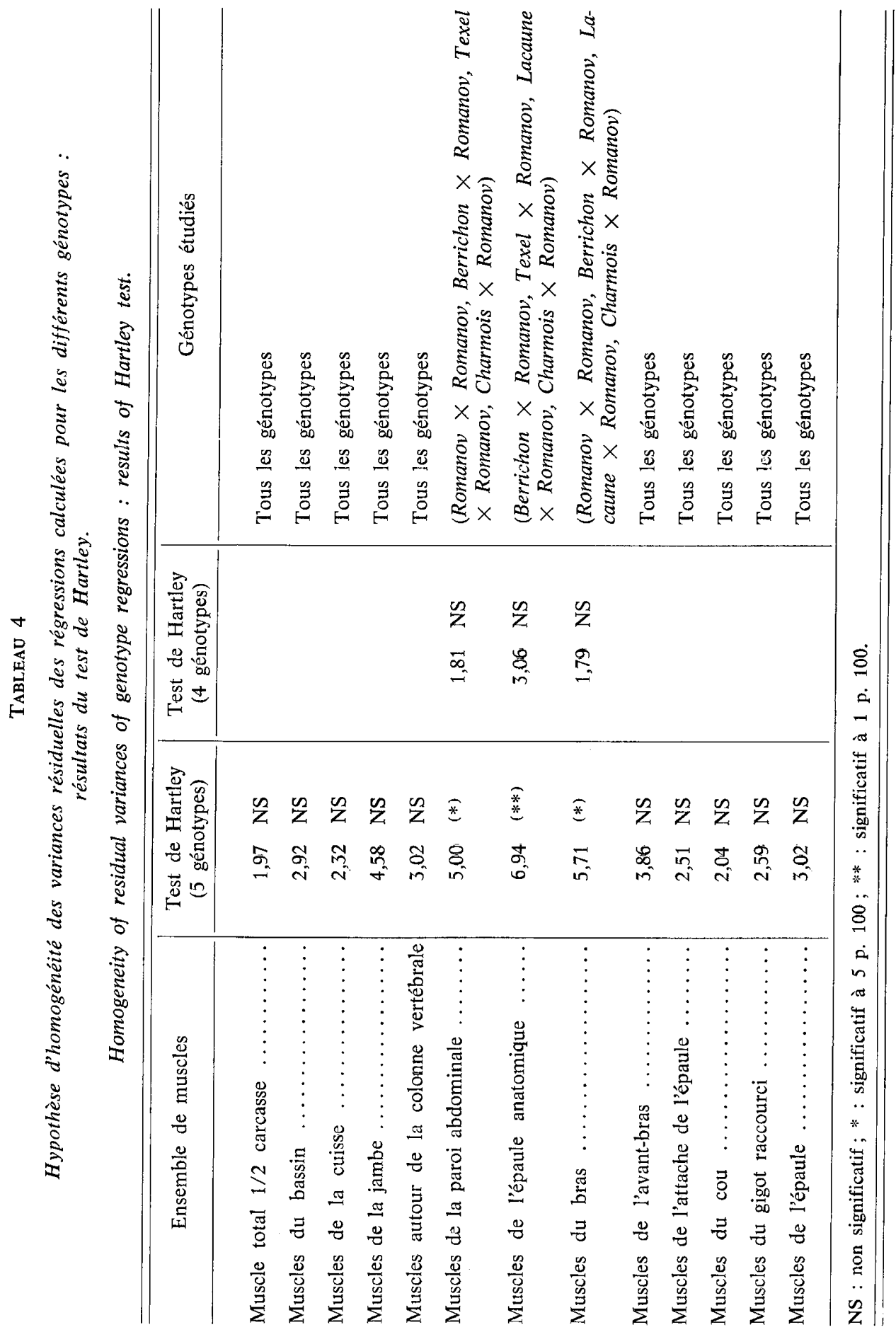




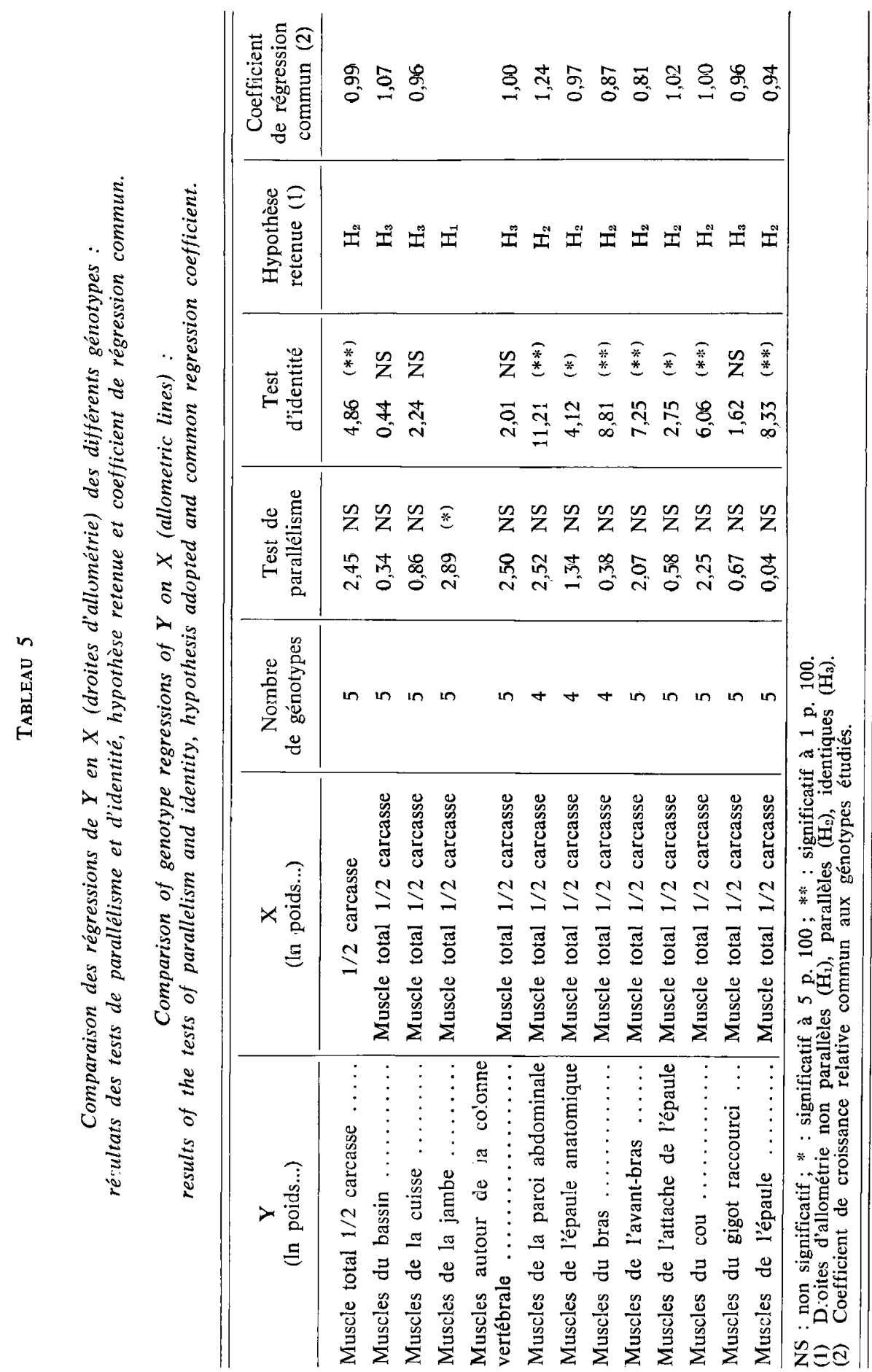




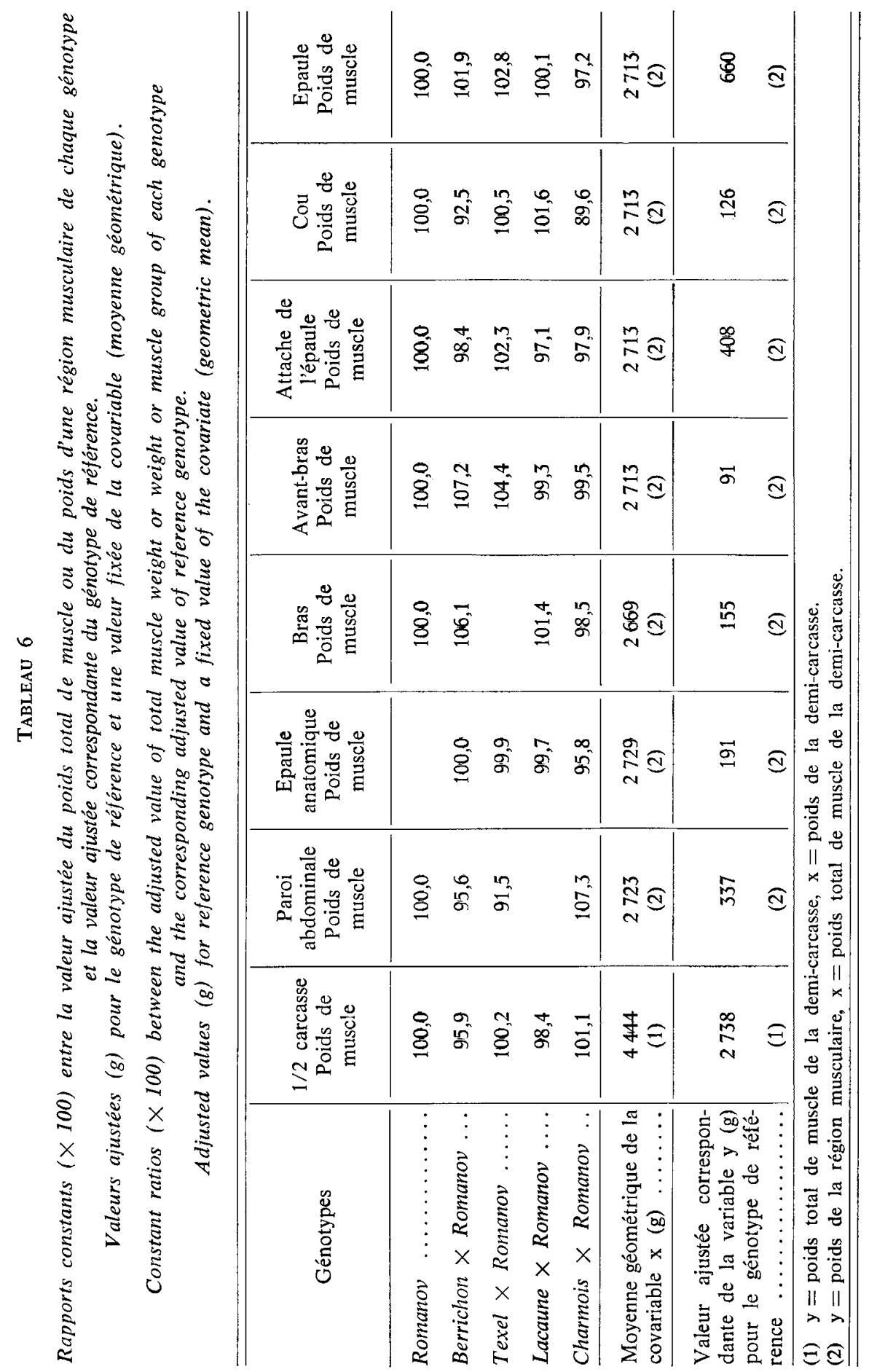


et al. (1980) montrent sur les mêmes agneaux que la moyenne, tous âges d'abattage confondus, de la proportion de tissu adipeux par rapport au poids vif des croisés Berrichon $\times$ Romanov est au moins supérieure de 1 p. 100 à celles des autres génotypes avec proportionnellement plus de dépôts de couverture quand on les compare aux Romanov et aux croisés Lacaune $\times$ Romanov. Dans une autre expérimentation de comparaison de plusieurs races de mâles utilisés en croisement, Flamant \& PerRET (1976) notent par dissection de l'épaule que les croisés Texel obtiennent le meilleur rapport muscle/gras, tandis que les Berrichons apparaissent les plus gras; l'état d'engraissement plus important des croisés Berrichons étant surtout net avec des mères croisées Romanov. L'adiposité plus élevée (ou plus précoce) des carcasses des croisés Berrichon $\times$ Romanov expliquerait donc en partie le moindre développement de la musculature que nous observons chez ces agneaux ( $-4,1$ p. 100 par rapport aux agneaux Romanov).

On peut rapprocher ce résultat de ceux obtenus par Fourie et al. (1970) pour les croisés Southdown $\times$ Romney, croisement couramment pratiqué en Nouvelle-Zélande pour la production d'agneaux de boucherie à partir de brebis Romney. Les carcasses des agneaux Southdown $\times$ Romney ont moins de muscle que les carcasses des agneaux de race pure Romney, respectivement $-2,2$ p. 100 et $-2,7$ p. 100 pour des carcasses de 20 et $30 \mathrm{~kg}$. Le développement du tissu adipeux, en particulier du gras de couverture, étant plus important dans les carcasses les agneaux croisés que dans les carcasses des agneaux Romney.

Les résultats que nous obtenons pour les autres croisements ne mettent pas en évidence de différences conséquentes dans l'importance du tissu musculaire à poids de carcasse égal.

\section{Poids des régions musculaires}

Il ressort des résultats donnés par le tableau 5 que l'hypothèse de parallélisme est rejetée $\left(\mathrm{H}_{1}\right)$ pour une seule région musculaire (muscles de la jambe). Les coefficients de croissance relative de cette région sont respectivement dans l'ordre où les génotypes sont présentés dans les tableaux $0,89,0,90,0,84,0,84$ et 0,85 . A l'exception de cette région l'évolution du poids de chaque région musculaire par rapport au poids total de muscle est décrite, pour les différents génotypes, par une même loi de croissance relative $\left(\mathrm{H}_{2}\right.$ ou $\left.\mathrm{H}_{3}\right)$. Les coefficients de croissance relative communs (tabl. 5) mettent bien en évidence le gradient de croissance classique inféro-supérieur du membre antérieur et du membre postérieur. On retrouve aussi un coefficient de croissance élevé $(1,24)$ pour les muscles de la paroi abdominale dont l'évolution est concomitante au développement du contenu digestif après le sievrage.

Dans le cas des muscles autour de la colonne vertébrale, du bassin, de la cuisse et du gigot raccourci, l'hypothèse d'identité n'est pas rejetée $\left(\mathrm{H}_{3}\right)$ ce qui implique l'absence d'effet du génotype sur l'importance relative de ces régions musculaires qui ont la plus grande valeur commerciale. Une étude de Boccard et al. (1961) sur des agneaux de poids et d'état d'engraissement similaires, appartenant à 3 types de races, avait déjà souligné que la forme du membre postérieur, appréciée par la mesure $F$ (longueur crosse-périnée), a une influence très limitée sur le poids et la répartition du tissu musculaire dans cette région à poids de carcasse constant.

Dans le cas des autres régions, l'hypothèse diidentité est rejetée $\left(\mathrm{H}_{2}\right)$ ce qui implique un effet significatif du génotype (effet additif). Il s'agit des régions mus- 
culaires de l'abdomen et de la partie antérieure de la caroasse (muscles de la paroi abdominale, de l'épaule anatomique, du bras, de l'avant-bras, de l'attache de l'épaule, du cou et de l'épaule). Les résultats de SEeBEck (1968) ne mettaient aussi en évidence, sur une partition moins précise du tissu musculaire (5 régions), un effet significatif du génotype que pour deux régions de la partie antérieure de la carcasse (cou et thorax).

Les valeurs obtenues pour ces dernières régions et les différents génotypes, exprimées en pourcentages du poids des régions musculaires des agneaux d'un génotype de référence (Romanov ou à défaut Berrichon $\times$ Romanov) sont données par le tableau 6. Les agneaux Berrichon $\times$ Romanov et Texel $\times$ Romanov dont les poids adultes sont les plus élevés ont moins de muscle dans la paroi abdominale (coefficient de croissance relative $=1,24)$ et plus de muscle dans le bras et l'avant-bras $(0,87$ et 0,81 ) que les agneaux de petit format : Romanov et Charmois $\times$ Romanov. Les différences de répartition trouvées dans ces trois régions, pour un même poids total de muscle, reflètent probablement des différences de maturité des agneaux. Si on tient compte de l'importance des régions musculaires dans la musculature totale, une différence parmi les plus importantes, trouvée pour la région abdominale quand on compare les croisés Texel aux agneaux Romanov, n'est que de $29 \mathrm{~g}$ pour un poids total de muscle de la demi-carcasse de $2723 \mathrm{~g}$ (1,1 p. 100). C'est aussi l'ordre de grandeur des différences maximales, entre génotypes, rapportées par JURY et al. (1977) dans les proportions des régions musculaires pour un poids total de muscle de la demi-carcasse de $2000 \mathrm{~g}$ et $4000 \mathrm{~g}$. ThOMPson et al. (1979 b) qui comparaient des agneaux plus lourds, entre 34 et $54 \mathrm{~kg}$, ne trouvent pas de différences significatives entre génotypes dans la répartition du tissu musculaire.

Les résultats obtenus pour les génotypes que nous étudions apparaissent donc conformes aux résultats déjà publiés sur les comparaisons d'autres génotypes. Ils confirment que les différences significatives trouvées dans la proportion des différentes régions musculaires, pour un même poids total de muscle, ont des conséquences économiques minimes. Cette tendance à l'homéostasie de la musculature est retrouvée dans d'autres études comparatives : comparaison de l'importance relative des principaux muscles d'agneaux de même race, élevés à deux vitesses de croissance très différentes (BocCARd \& Dumont, 1973), comparaison de génotypes de taurillons (BERg et al., 1978 ; Robelin \& GeAY, 1976).

\section{Conclusion}

Nous avons vu que l'analyse de covariance était particulièrement bien adaptée dans notre cas à la comparaison des caractéristiques musculaires des différents génotypes. Ce n'est pas un cas général. La dispersion des points expérimentaux autour des régressions peut remettre en cause certaines hypothèses de base comme la linéarité ou l'homogénéité des résiduelles entre génotypes (études comparatives de l'état d'engraissement des carcasses et de la répartition du tissu adipeux, par exemple).

Cette étude montre également la faible incidence, dans l'intervalle d'étude retenu, de la race du bélier utilisé en croisement sur l'importance de la musculature à poids de carcasse égal et sa répartition à poids de musculature égal, chez les agneaux 
croisés. Résultat qui est en accord avec la loi d'harmonie anatomique énoncée en 1960 par Boccard \& Dumont et retrouvée par KiRTon \& Pickering (1967).

Les différences significatives dans la répartition de la musculature sont trouvées essentiellement pour les régions de l'abdomen et de la partie antérieure de la carcasse qui sur le plan économique ne sont pas les plus intéressantes. Au moins dans certaines régions, elles paraissent imputables en grande partie à une différence dans la vitesse d'accession à la maturité des agneaux des différents génotypes.

Dans le cadre de cette étude, aucune analyse de la qualité bouchère des carcasses n'a été réalisée, l'effectif abattu à chaque stade étant trop réduit pour le faire. Il nous semble néanmoins possible de souligner que les différences entre génotypes pour ce caractère sont à rechercher dans des variations de conformation, d'état d'engraissement, de développement de l'ossature plutôt que dans des variations de répartition des masses musculaires pour un poids total de musculature fixé.

\section{Reçu pour publication en décembre 1981.}

\section{Remerciements}

Nous tenons à remercier M. Bibe et les membres de l'équipe de la S.A.G.A. de Toulouse pour leur contribution dans la réalisation de cette expérimentation. Nous remercions MM. BIBE et BocCARD pour les critiques et suggestions apportées à une première rédaction de cet article.

Cette étude a été réalisée dans le cadre de l’A.T.P. I.N.R.A. $\mathrm{n}^{\circ} 4158$.

\section{Summary}

Genetic differences in muscle weight and distribution, within 25-150 days of age, in Romanov, Berrichon $\times$ Romanov, Texel $\times$ Romanov, Lacaune $\times$ Romanov and Charmois $\times$ Romanov lambs

Eighty twin male lambs from 5 gerotypes (Romanov, Berrichon $\times$ Romanov, Texel $\times$ Romanov, Lacaune $\times$ Romanov, Charmois $\times$ Romanov) were slaughtered at different ages $(25,50,100$ and 150 days). Half carcass individual muscles were weighed and classified into 10 muscle groups in order to study the influence of sire breeds on the weight and distribution of total carcass muscle.

Basic hypotheses being verified, a model of covariance analysis was adopted to compare the allometric relationships established, for the different genotypes, between total muscle weight and carcass weight or between the weight of each muscle group and total muscle weight. This analysis enables us to adopt one of the three following hypotheses : nonparallel $\left(\mathbf{H}_{1}\right)$, parallel $\left(\mathrm{H}_{2}\right)$ or identical $\left(\mathbf{H}_{3}\right)$ allometric lines.

Except for muscles of the distal part of the pelvic limb $\left(\mathrm{H}_{1}\right)$, relative growth coefficients are homogenous among breeds $\left(\mathrm{H}_{2}\right.$ or $\left.\mathrm{H}_{3}\right)$ within the range of weights studied. At the same carcass weight, Berrichon $\times$ Romanov crossbreeds have least muscle and Charmois $\times$ Romanov crossbreeds have most muscle, respectively 95.9 and 101.1 when comparing them to Romanov lambs (100). The significant differences in muscle distribution at the same total muscle weight (especially muscle groups of the abdomen and fore part of the carcass) have minimal commercial implications. In certain cases, these differences seem to be due, to a great extent, to differences in maturity of the lambs from the genotypes under study. 


\section{Références bibliographiques}

BENEvent M., 1968. Guide pour la dissection et l'identification des principaux muscles de la carcass chez le Mouton. Ann. Biol. anim. Bioch. Biophys., 8, 147-193.

BenEvent M., 1971. Croissance relative pondérale postnatale, dans les deux sexes, des principaux tissus et organes de l'Agneau Mérinos d'Arles. Ann. Biol. anim. Bioch. Biophys., 11, 5-39.

Berg R.T., ANdersen B.B., Liboriussen T., 1978. Growth of bovine tissues. 2. Genetic influences on muscle growth and distribution in young bulls. Anim. Prod., 27, 51-61.

Boccard R., Dumont B.L., 1960. Etude de la production de la viande chez les ovins. II. Variation de l'importance relative des différentes régions corporelles de l'Agneau de boucherie. Ann. Zootech., 9, 355-363.

Boccard R., Dumont B.L., Le Guelte P., Arnoux J., 1961. Etude de la production de la viande chez les ovins. IV. Relation entre la forme et la composition du membre postérieur. Ann. Zootech., 10, 155-160.

Boccard R., Dumont B.L., 1973. Etede de la production de la viande chez les ovins. IX. Variation de l'organisation de la musculature de l'Agneau en fonction de la vitesse de croissance. Ann. Zootech., 22, 423-431.

Flamant J.C., Perret G., 1976. Le croisement et la production de viande d'apneaux, comparaison et sélection des races de mâles. Journées de la Recherche Ovine et Caprine, 110-128, I.N.R.A.-I.T.O.V.I.C., Paris, $1^{\mathbf{e r}}-2$ décembre 1976.

Fourie P.D., Kirton A.H., JuRY K.E., 1970. Growth and development of sheep. II. Effect of breed and sex on the growth and carcass composition of the Southdown and Romney and their cross. N.Z.J. agric. Res., 13, 753-770.

Jury K.E., FouriE P.D., Kirton A.H., 1977. Growth and development of sheep. IV. Growth of the musculature. N.Z.J. agric. Res., 20, 115-121.

Kirton A.H., Pickering F.S., 1967. Factors associated with differences in carcass conformation in lamb. N.Z.J. agric. Res., 10, 183-200.

Lohse C.L., Moss F.P., ButTerfeld R.M., 1971. Growth patterns of muscles of Merino sheep from birth to 517 days. Anim. Prod., 13, 117-126.

Masson J.P., 1976. Décisions sur le modèle de plan d'expérience à deux facteurs contrôlés. Revue de Statistique Appliquée, 24, 19-43.

McClelland T.H., Bonatti B., TaYlor C.S., 1976. Breed differences in body composition of equally mature sheep. Anim. Prod., 23, 281-293.

Perret G., Cournut J., 1975. Aptitudes des races ovines exploitées en France. Journées de la Recherche Ovine et Caprine, 2, 181-188, I.N.R.A.-I.T.O.V.I.C., Paris, 2-4 décembre 1975.

Prud'hon M., Teyssier J., Goussopoulos J., 1981. Etude des caractéristiques de croissance des principaux tissus, entre 25 et 150 jours, chez des agneaux appartenant à 5 génotypes : Romanov, Berrichon $\times$ Romanov, Texel $\times$ Romanov, Lacaune $\times$ Romanov et Charmois $\times$ Romanov. Options Médit., C.I.H.E.A.M. I.A.M.Z. - 81/Ill, 39-45.

Robelin G., Geay Y., 1976. Répartition des masses musculaires chez le jeune bovin mâle entier, et son évolution au cours de la période d'engraissement entre 8-9 et 16-17 mois. Ann. Zootech., 25, 273-279.

SEEbeCK R.M., 1968. A dissection study of the distribution of tissues in lamb carcasses. Proc. Aust. Soc. Anim. Prod., 7, 297-302.

Taylor C.S., Mason M.A., McClelland T.H., 1980. Breed and sex differences in muscle distribution in equally mature sheep. Anim. Prod., 30, 125-133.

Thompson J.M., AtKins K.D., Gilmour A.R., 1979 a. Carcass characteristics of heavyweight crossbred lambs. II. Carcass composition and partitioning of fat. Aust. J. agric. Res., 30, 1207-1214.

Thompson J.M., AtKins K.D., Gilmour A.R., 1979 b. Carcass characteristics of heavyweight crossbred lambs. III. Distribution of subcutaneous fat, intermuscular fat, muscle and bone in the carcass. Aust. J. agric. Res., 30, 1215-1221. 


\begin{abstract}
Annexe
Répartition des muscles de la carcasse dans les régions musculaires étudiées. Distribution of carcass muscles in the studied muscle groups.
\end{abstract}

1. Muscles du bassin

Fessier moyen, Fessier profond, Scansorius, Obturateurs (externe et interne), Jumeaux du bassin, Carré crural.
Glutaeus medius, Glutaeus accessorius, Glutaeus profondus, Obturatorius (externus et internus), Mm. gemelli, quadratus femoris.

2. Muscles de la cuisse

Couturier, Droit interne, Pectiné, Adducteur, Tenseur du fascia lata, Droit antérieur, Vaste externe, Vaste interne, Vaste intermédiaire, Sous-crural, Long vaste, Demi-tendineux, Demi-membraneux.
Sartorius, Gracilis, Pectineus, Adductor, Tensor fasciae latae, Rectus femoris, Vastus lateralis, vastus medialis, Vastus intermedius, Articularis genus, Biceps femoris et Adductor cruris cranialis, Semitendinosus, Semimembranosus.

\title{
3. Muscles de la jambe
}

Jumeaux (internes et externes), Perforé, Long péronier, Soléaire, Masse commune de la jambe, Poplité, Tibial antérieur, Extenseur propre du doigt externe, Fléchisseur interne des phalanges, Perforant, Tibial postérieur.
Gastrocnemius, Flexor digitorum superficialis, Peroneus longus, Soleus, Extensor digitorum longus, Popliteus, Tibialis cranialis, Extensor digitorum lateralis, Flexor digitorum longus, Flexor hallucis longus, Tibialis caudalis.

4. Muscles autour de la colonne vertébrale

Petit psoas, Grand psoas, Iliaque, Carré des lombes, Long dorsal, Long costal, Long épineux, Multifide dorso lombaire.
Psoas minor, Psoas major, Iliacus, Quadratus lumborum, Longissimus dorsi, Iliocostalis thoracis, Spinalis thoracis, Multifidus.

\section{Muscles de la paroi abdominale}

Peauciers, Rétracteur de la dernière côte, Petit dentelé, Grand oblique, Petit oblique, Droit de l'abdomen, Tranverse de l'abdomen.
Musculi cutanei, Retractor ultimae costae, Serratus dorsalis caudalis, Obliquus externus abdominis, Obliquus internus abdominis, Rectus abdominis, Transversus abdominis.

\section{Muscles de l'épaule anatomique}

Deltoïde, Sus-épineux, Sous-épineux, Petit rond, Grand rond, Coraco-brachial, Sous-scapulaire.
Deltoideus, Infraspinatus, Supraspinatus, Teres minor, Teres major, coracobrachialis, Subscapularis. 
7. Muscles du bras

Biceps, Brachial antérieur, Long anconé, Anconé externe, Anconé interne, Petit anconé, Anconé accessoire du Grand dorsal.
Biceps brachii, Brachialis, Caput longum tricipitis brachii, Caput laterale tricipitis brachii, Caput mediale tricipitis brachii, Anconoeus, Tensor fasciae antebrachii.

\section{Muscles de l'avant-bras}

Extenseur antérieur du métacarpe, Extenseur propre du doigt interne, Extenseur propre du doigt externe, Extenseur commun des doigts, Cubital externe, Cubital interne, Grand palmaire, Perforé, Perforant.
Extensor carpi radialis, longus et brevis, Extensor digitorum lateralis, Extensor digitorum communis, Extensor carpi ulnaris, Flexor carpi ulnaris, Flexor carpi radialis, Flexor digitorum superficialis, Flexor digitorum profondus.

\section{Muscles de l'attache de l'épaule}

Brachio-céphalique, Omo-trachélien, Trapèze, Rhomboïde, Grand dorsal, Grands dentelés, Pectoral descendant, Pectoral transverse, Pectoral ascendant.
Brachiocephalicus, Omotransversarius, Trapezius, Rhomboïdeus, Latissimus dorsi, Serratus ventralis cervicis et Serratus ventralis thoracis, Pectoralis descendens, Pectoralis transversus, Pectoralis ascendens.

10. Muscles du cou

Sterno-céphalique, Sterno-hyoïdien, Sterno-thyroïdien, Scalène, Splénius, Grand complexus, Petit complexus, Transversaire du cou, Long du cou...
Sternocephalicus, Sternohyoüdeus, Sternothyroïdeus, Scalenus, Splenius, Semispinalis capitis, Mm. longissimus capitis et longissimus atlantis, Longissimus cervicis, longus colli...

\section{Muscles du gigot raccourci}

Muscles du bassin, Muscles de la cuisse, Muscles de la jambe.

\section{Muscles de l'épaule}

Muscles de l'épaule anatomique, Muscles du bras, Muscles de l'avant-bras, Trapèze, Rhomboïde, Grand dorsal, Grands dentelés. 